\title{
Application of localized reactivity index in combination with periodic DFT calculation to rationalize the swelling mechanism of clay type in- organic material
}

\author{
ABHIJIT CHATTERJEE \\ Accelrys K K, Nishishinbashi TS Bldg. 11F. 3-3-1 Nishishinbashi, Minato-ku, Tokyo 105-0003, Japan \\ e-mail: achatterjee@accelrys.com
}

\begin{abstract}
Clays are layered alumino-silicates. Clays swell and expand in aqueous solution. This property governs the usage of these materials in synthesis of nano-composites and is a source of many of its catalytic applications. We used both localized and periodic calculations within the realm of density functional theory (DFT) on a series of monovalent $\left(\mathrm{Li}^{+}, \mathrm{Na}^{+}, \mathrm{K}^{+}, \mathrm{Rb}^{+}, \mathrm{Cs}^{+}\right)$, and divalent $\left(\mathrm{Mg}^{2+}, \mathrm{Ca}^{2+}, \mathrm{Sr}^{2+}, \mathrm{Ba}^{2+}\right)$ cations, to monitor their effect on the swelling of clays. The activity order obtained for the exchangeable cations among all the monovalent and divalent series studied is: $\mathrm{Mg}^{2+}>\mathrm{Ca}^{2+}>\mathrm{Sr}^{2+}>\mathrm{Ba}^{2+}>\mathrm{Cs}^{+}>$ $\mathrm{Rb}^{+}>\mathrm{Na}^{+}>\mathrm{Li}^{+}>\mathrm{K}^{+}$. We have studied two types of clays, montmorillonite and beidellite, with different surface structures and with/without water using periodic calculations. We have calculated the layer spacing at the first, second and third hydration shells of exchangeable cation, to compare with the experimental $d$-spacing values to correlate with humidity. A novel quantitative scale is proposed in terms of the intermolecular relative nucleophilicity of the active cation sites in their hydrated state through Fukui functions using hard-soft acid base (HSAB) principle. Finally, a swelling mechanism is proposed. This is a unique study where a combination of periodic and localized calculations has been performed to validate the capability of reactivity index calculations in material designing.
\end{abstract}

Keywords. Localized reactivity index; periodic DFT calculations; swelling mechanism; inorganic clays; interlayer cations.

\section{Introduction}

Clays are lamellar alumino-silicates with a large variety of physicochemical properties, such as swelling, adsorption, surface acidity, ion exchange etc. Smectites are 2:1 dioctahedral structural units of clay, with one octahedral layer sandwiched between two tetrahedral layers. Montmorillonite and beidellite are members of this 2: 1 dioctahedral smectite family. They share the common feature that two tetrahedral sites sandwich a sheet of octahedrally coordinated metal ions. Substitution of a bivalent metal ion for octahedral aluminum in montmorillonite and substitution of a trivalent metal ion for tetrahedral silicon in beidellite results in a net negative layer charge, and interaction with positive ions (exchangeable cation) to form an interlayer hydrated phase. There exists a high repulsive potential on the surface resulting from isomorphous substitution. Interlayer cation and charged clay surfaces interact strongly with polar solvents. As a result 2:1 clays expand in the presence of water in solution. This process is known as crystalline swelling. ${ }^{1}$ The extent of swelling is controlled by a balance be- tween relatively strong swelling forces, due to the hydration potential of the interlayer cations, charge sites, electrostatic forces of attraction between the positively charged interlayer cation ${ }^{2}$ and negatively charged 2:1 phyllosilicate layer. The amount of water adsorbed by smectites is a function of the interlayer cations, is shown by Cuardos $e t a l^{3}$ using different experimental techniques such as BET surface area measurements, X-ray diffraction (XRD) and thermogravimetric (TG) analysis. The equilibrium hydration state of a clay in integer-layer hydrates ${ }^{4,5 a}$ regime is known to be a function of the magnitude and location of the clay layer charge, the applied pressure, the temperature, the interlayer ion identity and the water chemical potential as determined by vapour pressure or solution ionic strength using molecular dynamics. A recent experimental study probed the influence of layer charge on the hydration of the external surface of expandable 2:1 phyllosilicates. ${ }^{5 \mathrm{~b}}$ The model assumes that interlayer volume controls interlayer hydration and the number of cation/charge sites on external surfaces controls hydration of external surfaces. There is consensus about the fact that swelling occurs through 
distinct steps forming one-, two-, and three-layer hydrates. ${ }^{6}$ Many computer simulations have been directed to understand the swelling of clays or to study the clay-water interface. ${ }^{7,8-11}$

In our earlier study ${ }^{12,13}$ we rationalized the structure property relationship in montmorillonite clays and observed that these hydroxyl groups can be expected to play a crucial role in the catalytic activity of dioctahedral clays. Pearson proposed the global HSAB principle ${ }^{14}$ and the principle of maximum hardness $(\mathrm{PMH})^{15}$ which states that, for a constant external potential, the system with the maximum global hardness is most stable. Some DFT-based local properties, e.g. Fukui functions and local softness, ${ }^{16}$ have already been used for reliable predictions in various types of electrophilic and nucleophilic reactions. Now, it is known that the reactivity of a chemical species depends on the solvent associated around the molecules. ${ }^{17}$ Generally, when compared to gas-phase calculations, the solvent environment alters the charge distribution of molecules and there is thus an increase in the dipole moment of the molecules. In addition to that, water enhances the intrinsic reactivity of polar molecules towards nucleophilic or electrophilic attack. ${ }^{17}$ In a recent study, ${ }^{18}$ we have used both localized reactivity index and periodic calculation on a series of monovalent and divalent cation in their monohydrated from to monitor their effect on the swelling of clays, and have proposed a qualitative scale.

In the present study, we attempt to extend the study for the same series of metal cations in the presence of higher humidity to monitor the feasibility of two-layer or three-layer hydration using both reactivity index and periodic density functional calculation. We compare the hydration behaviour for all these cations and propose a plausible mechanism of hydration. We have calculated the layer spacing at the first, second and third hydration shell of exchangeable cation for comparison with the experimental $d$-spacing values to correlate with humidity. A novel quantitative scale is proposed by the relative nucleophilicity of the active cation sites in their hydrated state through Fukui functions within the hard soft acid base (HSAB) principle.

\section{Theory}

In density functional theory, hardness $(\eta)$ is defined as, ${ }^{14}$

$$
\eta=1 / 2\left(\delta^{2} E / \delta N^{2}\right) v(r)=1 / 2(\delta \mu / d N)_{v},
$$

where, $E$ is the total energy, $N$ is the number of electrons of the chemical species and $\mu$ is the chemical potential.

The global softness, $S$, is defined as the inverse of the global hardness, $\eta$,

$$
S=1 / 2 \eta=(\delta N / \delta \mu)_{v} .
$$

Using the finite difference approximation, $S$ can be approximated as

$$
S=1 /(\mathrm{IE}-\mathrm{EA}),
$$

where, IE and EA are the first ionization energy and electron affinity of the molecule respectively.

The Fukui function $f(r)$ is defined as mentioned, ${ }^{18}$

$$
f(r)=[\delta \mu / \mathrm{d} v(r)]_{N}=[\delta \rho(r) / \delta N]_{v} .
$$

The function $f$ is thus a local quantity, which has different values at different points in the species, $N$ is the total number of electrons, $\mu$ is the chemical potential and $v$ is the potential acting on an electron due to all the nuclei present.

The local softness $s(r)$ can be defined as

$$
s(r)=(\delta \rho(r) / \delta \mu)_{v} .
$$

Equation (3) can also be written as

$$
s(r)=[\delta \mu(r) / \delta N] v[\delta N / \delta \mu]_{v}=f(r) S .
$$

Thus, local softness contains the same information as the Fukui function $f(r)$ plus additional information about the total molecular softness, which is related to the global reactivity with respect to a reaction partner, as stated in the HSAB principle. Atomic softness values can easily be calculated by using (4), namely:

$$
\begin{aligned}
& s_{x}^{+}=\left[q_{x}(N+1)-q_{x}(N)\right] S, \\
& s_{x}^{-}=\left[q_{x}(N)-q_{x}(N-1)\right] S, \\
& s_{x}^{0}=S\left[q_{x}(N+1)-q_{x}(N-1)\right] / 2 .
\end{aligned}
$$

\section{Computational methodology and model}

The software package CASTEP (Cambridge Serial Total Energy Package), which has been described elsewhere, ${ }^{19,20}$ and associated programs for symmetry analysis were used for the calculations. Becke- 
Perdew parameterization ${ }^{21,22}$ of the exchange-correlation functional, which includes gradient correction (GGA), was employed. The pseudo potentials are constructed from the CASTEP database. The screening effect of core electrons is approximated by LDA, while the screening effect for valence electrons is approximated by GGA. To obtain equilibrium structures for a given set of lattice constants, ionic and electronic relaxations were performed using the adiabatic or 'Born-Openheimer' approximation, where the electronic system is always in equilibrium with the ionic system. Relaxations were continued until the total energy had converged. In the present calculations kinetic cut-off energies between 600 and $1500 \mathrm{eV}$ have been used. The Monkhorst-Pack scheme ${ }^{23}$ was used to sample the Brillouin zone. The calculations were restricted to one special $\mathrm{K}$ point in the Brillouin zone, placed at $(0 \cdot 0,0 \cdot 0$, and $0 \cdot 0)$.

In the present study, all localized cluster calculations have been carried out with $\mathrm{DFT}^{24}$ using the $\mathrm{DMOL}^{3}$ code of Accelrys Inc. A gradient corrected functional BLYP ${ }^{25,26}$ and DNP basis $\operatorname{set}^{27}$ was used through out the calculation. Basis set superposition error (BSSE) was also calculated for the current basis set in non-local density approximation (NLDA). Single point calculations of the cation and anion of each molecule, at the optimized geometry of the neutral molecule were also carried out to evaluate Fukui functions, global and local softness. The condensed Fukui function and atomic softness were evaluated using (3) and (5) respectively. The gross atomic charges were evaluated by using the technique of electrostatic potential (ESP) driven charges.

The ideal formula of the clay montmorillonite, a member of $2: 1$ dioctahedral smectite family, is $\left(M_{x}^{+}\right.$, $\left.n \mathrm{H}_{2} \mathrm{O}\right)\left(\mathrm{Al}_{4-x} \mathrm{Mg}_{x}\right) \mathrm{Si}_{8} \mathrm{O}_{20}(\mathrm{OH})_{4},{ }^{12}$ where $x=(12-a-b)$ is the layer charge, and $M$ is the balancing cation which is either monovalent or divalent. The desired structures of montmorillonite and beidellite have been generated from the structure of well-defined pyrophyllite. ${ }^{28}$ They have the formula $\mathrm{MSi}_{8} \mathrm{Al}_{3} \mathrm{MgO}_{20}$ $(\mathrm{OH})_{4}$ and $\mathrm{MAlSi}_{7} \mathrm{Al}_{4} \mathrm{O}_{20}(\mathrm{OH})_{4}$ respectively. Hydrated phase calculations were performed with the minimum energy structures of the clays at the unhydrated phase. The interlayer structure was held fixed and the interlayer spacing was increased to accommodate the water molecule (figure 1). Unhydrated respective clay structures were minimized using two steps: (1) the tetrahedral layers were allowed to relax with octahedral ions were kept fixed, and (2) all the ions were relaxed simultaneously relative to a fixed octahedral ion (magnesium or aluminum) until the total energies were converged. In both steps the interlayer cation is relaxed. For the hydrated phases we relaxed the cation water and the interlayer space, the lower part of the structure was kept fixed to compromise between CPU cost and accuracy.

The water complexes for the individual cations were generated from the optimized periodic structure. The geometries of the localized cations were isolated and then relaxed again for each neutral cationic and anionic form for the calculation of reactivity indices.

\section{Results and discussion}

This is known that at increasing humidity, smectites adsorb water vapor and form one-, two- and threelayer hydrates. The general swelling model within the interlayer of the smectite clay is shown in Figure 2. Bonded water molecules are present as shown in the figure while non-bonded water molecules, which act as bulk water, are not shown here for visual clarity. We first compared the structure of two types of smectite, namely montmorillonite and biedellite. For beidellite, the negative charge associated with the tetrahedral layer substitution can be distributed over just three oxygens of one tetrahedron. This means that the water experiences a localized negative charge, which results in strong binding of water and interlayer cations.

Water experiences a more diffuse negative charge associated with octahedral substitution, for montmorillonite. It is well observed that in terms of ionic radii the cations studied are in the order $\mathrm{Cs}^{+}>\mathrm{Rb}^{+}>\mathrm{K}^{+}>$ $\mathrm{Ba}^{+2}>\mathrm{Sr}^{+2}>\mathrm{Na}^{+}>\mathrm{Ca}^{+2}>\mathrm{Li}^{+}>\mathrm{Mg}^{+2}$.

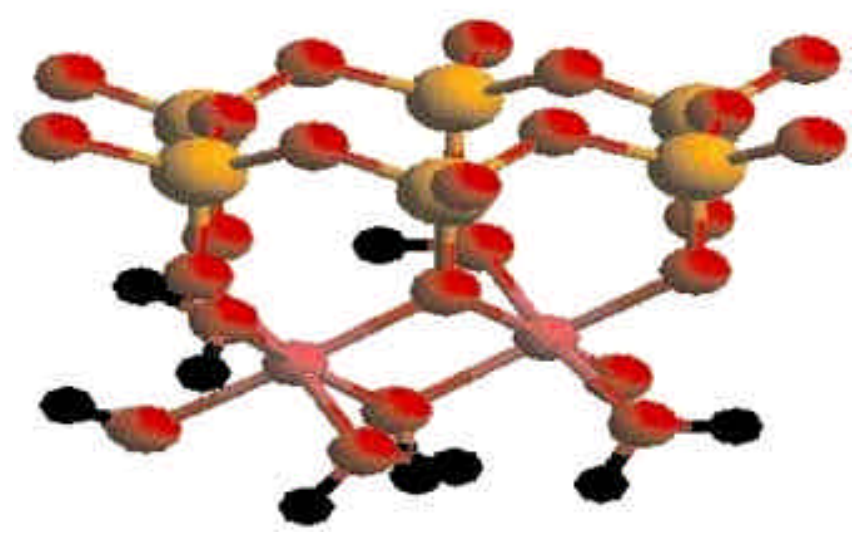

Figure 1. The structure representing 2:1 clay structure, the top layer consists of tetrahedral $\mathrm{Si}$ and the bottom of octahedral Al. 
(a)

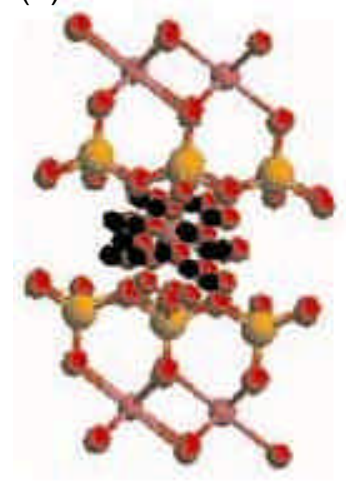

(b)

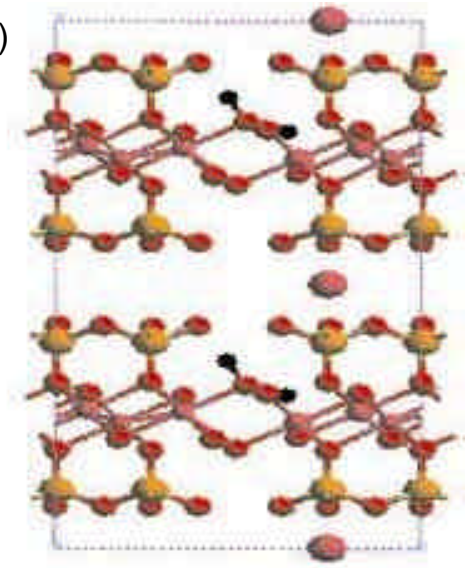

Figure 2. (a) Smectite swelling model where the central cation is hydrated with water molecules. (b) Model showing the interlayer cation inside the clay layers.

Depending on the extent of increment in basal spacing between two smectite sheets, two types of swelling mechanism are proposed; (1) crystalline swelling and (2) osmotic swelling. Crystalline swelling occurs for a situation with monolayers of water adsorption around the cations, held by hydrogen bonding to the hexagonal network of oxygen atoms. This could result in an increase of $d$-spacing in the range of about 10 $20 \AA$. Osmotic swelling is a phenomenon when interlayer spacing increases abruptly with water content to $30-40 \AA$ due to the interaction of the layer. We have explored the clay materials for their unique catalytic property. ${ }^{18}$ Here we have chosen models of montmorillonite- and beidellite-type clays with structural formula $\mathrm{MSi}_{8} \mathrm{Al}_{3} \mathrm{MgO}_{20}(\mathrm{OH})_{4}$ and $\mathrm{MAlSi}_{7} \mathrm{Al}_{4} \mathrm{O}_{20}(\mathrm{OH})_{4}$ respectively, and optimized the structure in their unhydrated forms. The internal coordinates matches with the available experimental values. The deviation is mostly at the basal oxygen atoms of the tetrahedral layer due to the rotation of the $\mathrm{SiO}_{4}$ tetrahedral by $13.2^{\circ}$ and the tilt by $4.6^{\circ}$ on the average. It is observed that for montmorillonite the hydroxyl hydrogen attached with the octahedral aluminum makes an angle of $24 \cdot 12^{\circ}$ with the $a-b$ plane. We first optimized the montmorillonite/beidellite structure with different monovalent and divalent exchangeable cations. Then we added water around the cation centre starting from first hydration shell to third hydration shell. We have used a cut off distance for this hydration shell, which is $2 \AA$ for one layer, $3 \AA$ for two layers and $4 \AA$ for three layers of hydration. We have calculated the cell parameters after hydration to rationalize the swelling phenomenon.

The model used for calculation is shown in figure 3 . Water molecules are added until there is a constant rise in the stabilization energy to allow the maximum number of water molecule to reside in the first hydration shell. For monovalent cations the number of water molecules surrounding the cations is 5 and that for bivalent cations is 3 in the first hydration shell as observed at the forced boundary condition. This result matches with our earlier Grand Canonical Monte Carlo GCMC simulation. ${ }^{29}$

The general trend exhibits that for all the cases there is a swelling along $c$-direction after hydration except for potassium. This can be explained by the fact that, in these types of clays one tetrahedral sheet of one unit layer is adjacent to another tetrahedral sheet of another layer. The oxygen atoms here are opposite to one another and the bonding between the layers is weak. Also there exists a high repulsive potential on the surface of layers resulting form isomorphous substitution. These factors contribute to the increase of the unit cell in c-direction due to the penetration of water. The results obtained for both the smectite type montmorillonite and beidellite studied are shown in figures $4 \mathrm{a}$ and $\mathrm{b}$ respectively. In both the cases we have plotted the layer spacing with respect to the exchangeable cation studied. It is observed for potassium that the layer even shrinks after the first hydration in both the smectite varieties. The swelling resulted from the first hydration is more pronounced for monovalent interlayer cations compared to the bivalent cations. The trend changes for the layer spacing when calculated for higher layer of hydration - two layers and three layers respectively. The result shows a better trend for the beidellite variety, as the layer spacing increases or decreases in order with few exceptions. We have observed that the layer expansion is less for monovalent interlayer cation species compared to bivalent cations. This is especially observed in case of three layer hydration. The results can be explained in terms of the experimental proposition that as the layer charge in case of beidellite is more localized, the resulting expansion due to swelling forces is less compared to the diffused situation in montmorillonite, as the layer charge is generated from the octahedral substitution. The lower expansion observed for the monovalent cations in their three-layer hydration model validates the model of Ransom and Helgesson. ${ }^{30}$ In their model for sodium-type bentonite clay based on thermodynamic calculation they treated the $15 \AA$ hydrate and the anhydrate as the end members 

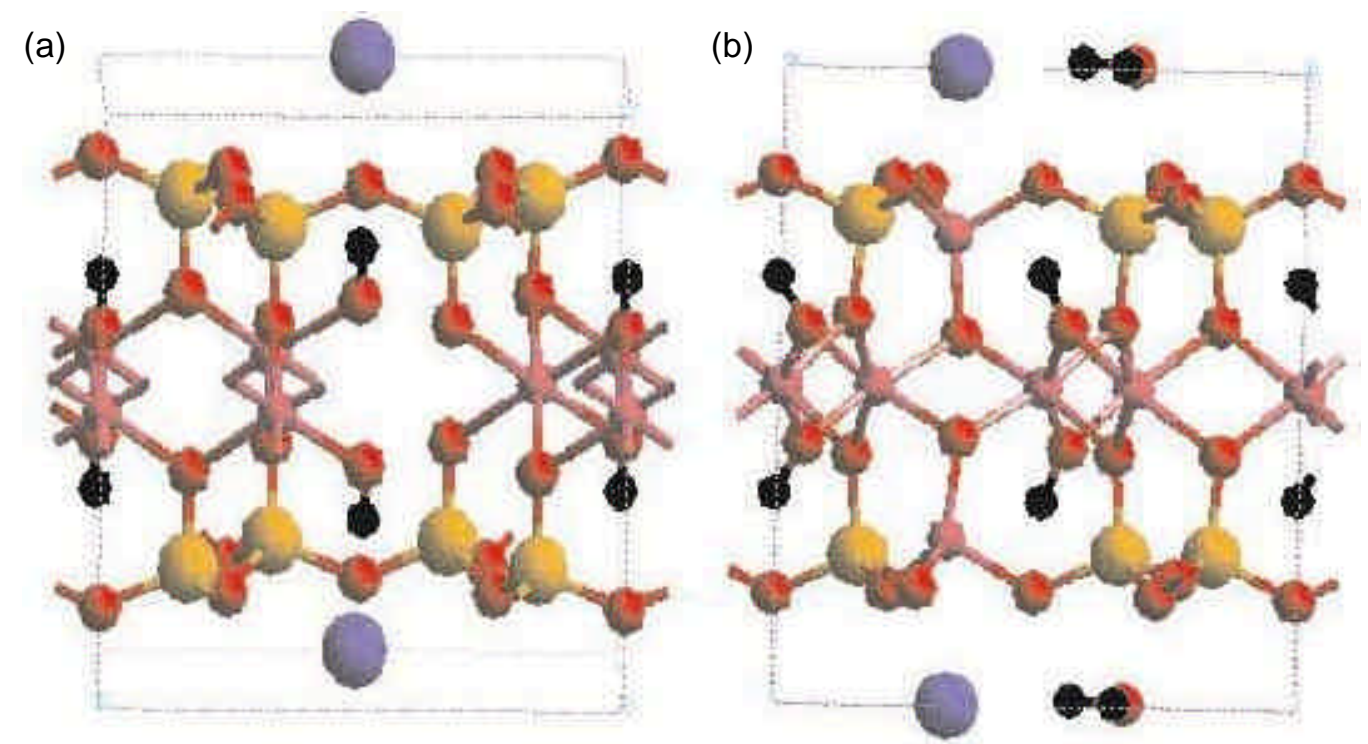

Figure 3. (a) Optimized structure of the sodium cation over montmorillonite type clay structure, where octahedral aluminum is substituted by magnesium. (b) Model clay structure with cation to show water addition inside a radius of $2 \AA$ as an example.
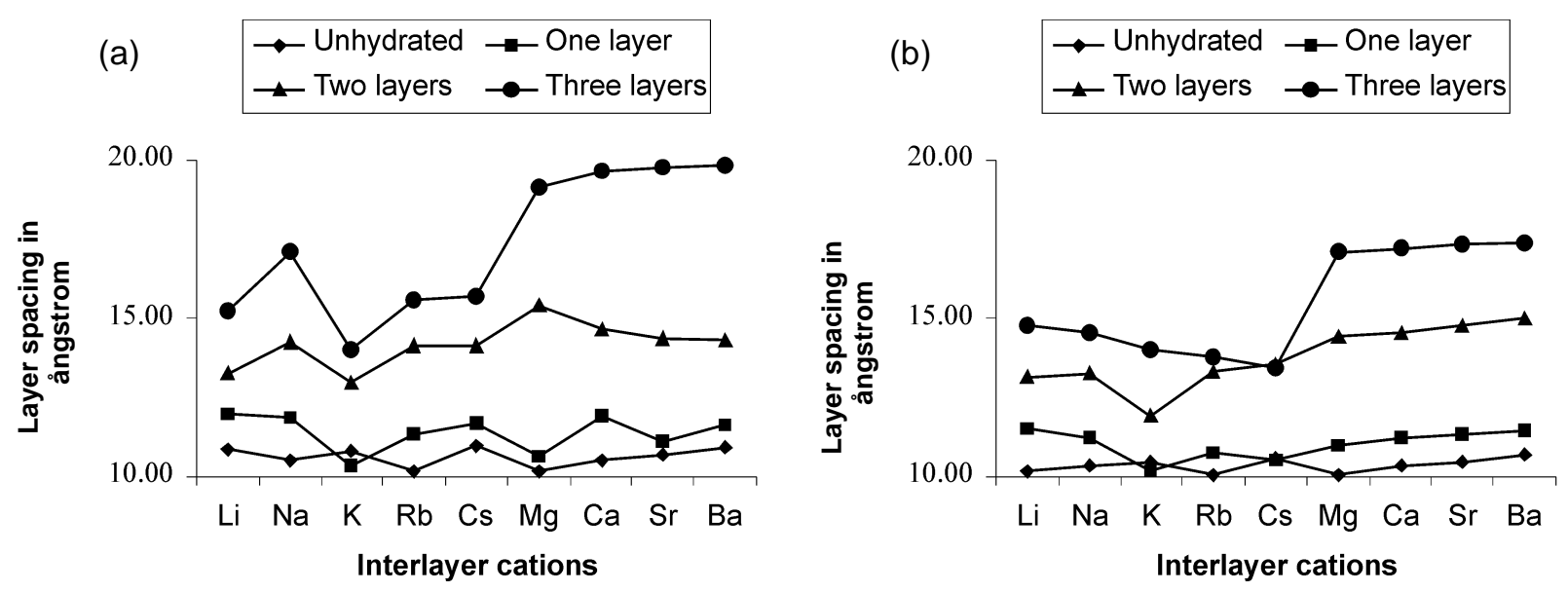

Figure 4. The effect of layer spacing in (a) montmorillonite and (b) beidellite in presence of different interlayer cations with varying layers of hydration.

of a solid solution series and chose to disregard the $19 \AA$ A regimes because they assumed that water content has thermodynamic properties about the same as those of bulk water. This effect is contradicted by Basett et al, ${ }^{31}$ where they show that this $19 \AA$ Aregime holds good for bivalent metal cations, e.g. $\mathrm{Ca}$ and $\mathrm{Mg}$. The order of swelling in terms of layer spacing, as observed for one-, two- and three-layer hydration respectively for all the interlayer cations studied, is as follows: $\mathrm{Li}>\mathrm{Ca}>\mathrm{Na}>\mathrm{Cs}>\mathrm{Sr}>\mathrm{Rb}>\mathrm{Ba}>$ $\mathrm{Mg}>\mathrm{K} ; \mathrm{Mg}>\mathrm{Ca}>\mathrm{Ba}>\mathrm{Sr}>\mathrm{Na}>\mathrm{Cs}>\mathrm{Rb}>\mathrm{Li}>$ $\mathrm{K} ; \mathrm{Sr}>\mathrm{Ba}>\mathrm{Ca}>\mathrm{Mg}>\mathrm{Na}>\mathrm{Cs}>\mathrm{Rb}>\mathrm{Li}>\mathrm{K}$. The order is given only for montmorillonite as there are no experimental results available for comparison with beidellite. It is observed that experimentally ${ }^{3}$ in terms of $d$-spacing, the order is $\mathrm{Mg}>\mathrm{Ca}>\mathrm{Sr}>$ $\mathrm{Rb}>\mathrm{Ba}>\mathrm{Li}>\mathrm{Na}>\mathrm{Cs}>\mathrm{K}$. This order is obtained at a fixed humidity and so the expansions are related to the number of water layer present at that humidity. Our layer spacing results are therefore only a partial match with experimental findings, and can reproduce the lowest expansion of potassium as observed by experiment very well. Whereas, considering the humidity of $30 \%$, the energetic characteristic obtained from the periodic calculations reproduces the trend of experiment. This trend is therefore mostly for 

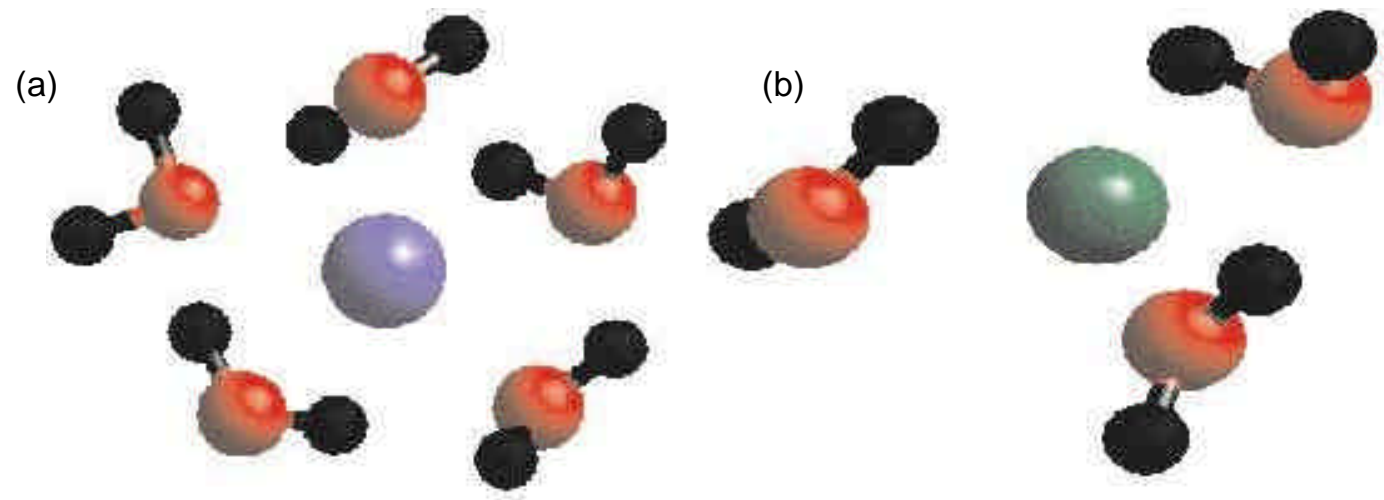

Figure 5. The model for (a) monovalent cation mono hydrated complex with five water molecules and (b) bivalent cation complex with three water molecules as obtained from periodic calculation.

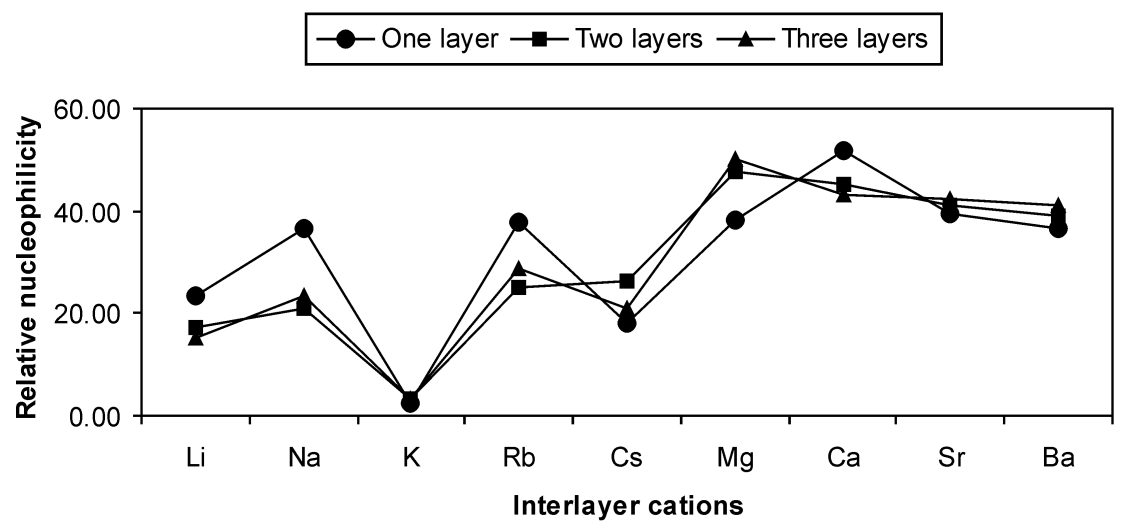

Figure 6. The plot of relative nucleophilicity with respect to the interlayer cations.

the monolayer hydration, the effect for increased humidity condition cannot therefore be obtained though the periodic calculations. We cannot see any pronounced difference between monovalent and divalent cations to account for the difference in swelling phenomenon at higher humidity. This shows that the bulk calculation is unable to match the experimental trend of swelling. Hence, it appears that it would be worth while to monitor the localized water environment around the cation, which may then be able to reproduce the experimental trend, and predict the mechanism of multilayer hydration situation for a specific exchangeable cation. We need to answer why one set of cations (bivalent) has shown increased swelling at the same humidity when the other cation (monovalent) cannot. We have performed localized calculations with both monovelent and divalent cations in their solvated forms to study the cationwater complex. We have taken out the cation-water complex as obtained from the optimized periodic cal- culation of the specific hydration model for a cation and performed a localized calculation to compare the reactivity index at that specific configuration using the method described elsewhere. ${ }^{13}$ The models and the results are shown in figures 5 and 6 respectively.

The results show that in terms of relative nucleophilicity (which is the ratio of nucleophilicity of a particular atom centre) the order of activity for one-, two- and three-water scenario for montmorillonite is as follows: $\mathrm{Ca}>\mathrm{Sr}>\mathrm{Mg}>\mathrm{Rb}>\mathrm{Ba}>\mathrm{Na}>\mathrm{Li}>$ $\mathrm{Cs}>\mathrm{K} ; \mathrm{Mg}>\mathrm{Ca}>\mathrm{Sr}>\mathrm{Ba}>\mathrm{Cs}>\mathrm{Rb}>\mathrm{Na}>\mathrm{Li}>$ $\mathrm{K} ; \mathrm{Mg}>\mathrm{Ca}>\mathrm{Sr}>\mathrm{Ba}>\mathrm{Rb}>\mathrm{Na}>\mathrm{Cs}>\mathrm{Li}>\mathrm{K}$. This activity order is in close resemblance with the experimental $d$-spacing results. We have as well seen from the optimized geometry of the cation water complex, the most stable bivalent hydrate complex $\mathrm{Mg}$ is surrounded by 6 water molecules in a twolayer hydration with a cut-off radius of $3 \AA$. One of the $\mathrm{H}$ atoms of each water molecule is pointed directly towards an oxygen atom of the adjacent clay 
layer. This also confirms that a tetrahedral network of water molecules similar to pure ice or water does not form due to the complicated influence of clay sheets and the interlayer cation. The difference in behaviour of monovalent and divalent cation is therefore mainly due to localized cation-water interactions.

\section{Conclusions}

In this communication we have studied a range of monovalent and divalent interlayer cations and their interactions with water during the swelling process for $2: 1$ dioctahedral smectite clay with special emphasis on montmorillonite for all possible 1-, 2- and 3-hydration layer situations. A quantitative order of activity comparable with the experimental $d$-spacing is obtained. It is observed that at low hydration the water molecules try to squeeze themselves in a flat orientation and the trend is quite random depending on the location of layer charge. With increase in water content the water molecules form stretched $\mathrm{H}$-bonds with the clay surface and other water molecules. $\mathrm{Mg}$ proves to be the best cation for swelling with higher water and $\mathrm{K}$ always remains bound to the clay surface and acts as a swelling inhibitor.

\section{References}

1. Sposito G and Prost R 1982 Chem. Rev. 82554

2. Laird D A 1996 Clays Clay Miner. 44553

3. Cuadros J 1997 Am. J. Sci. 297829

4. Shroll R M and Smith D E 1999 J. Chem. Phys. 111 9025

5. (a) Kutter S, Hansen J P, Sprik M and Boek S $2000 \mathrm{~J}$. Chem. Phys. 112 311; (b) Laird D A 1999 Clays Clay Miner. 47630

6. Brindley G W and Brown G 1980 Crystal structures of clay minerals and their X-ray identification (London: Mineral. Soc.)

7. Hensen E J M and Smit B 2002 J. Phys. Chem. B106 12664 , and references therein
8. Eypert-Bliason C, Michot L J, Humbert B, Pelletier M, Villieras F and Caillerie J B 2002 J. Phys. Chem. B106 730

9. Marry V and Turq P J 2003 J. Phys. Chem. B107 1832

10. Boek E S and Sprik M J. Phys. Chem. 2003 B107 3251

11. Tambach T J, Hensen E J M and Smit B $2004 \mathrm{~J}$. Phys. Chem. B108 7586

12. Chatterjee A, Iwasaki $\mathrm{T}$, Hayashi $\mathrm{H}$, Ebina $\mathrm{T}$ and Torri K 1998 J. Mol. Catal. A136 195

13. Chatterjee A, Iwasaki T and Ebina T 2000 J. Phys. Chem. A104 8216

14. Pearson R G 1983 J. Am. Chem. Soc. 1057512

15. Pearson R G 1987 J. Chem. Edu. 64561

16. Geerlings P, De Proft F and Langenaekar W 2003 Chem. Rev. 103 1793, and references therein

17. Sivanesan D, Amutha R, Subramanian V, Nair B U and Ramasami T 1999 Chem. Phys. Lett. 308223

18. (a) Chatterjee A et al 2003 J. Chem. Phys. 118 10212; J. Mol. Graphics Modelling 200322 93; 2004 J. Chem. Phys. 1203414

19. Teter M P, Payne M C and Allen D C 1989 Phys. Rev. B40 12255

20. Payne M C, Teter M P, Allan D C, Arias T A and Johannopoulos J D 1992 Rev. Modern Phys. 641045

21. Perdew J P 1986 Phys. Rev. B33 8822

22. Becke A D 1988 Phys. Rev. A33 3098

23. Monkhorst H J and Pack J D 1976 Phys. Rev. B13 5188

24. Kohn W and Sham L J 1965 Phys. Rev. A140 1133

25. Becke A J 1988 Chem. Phys. 882547

26. Lee C, Yang W and Parr R G 1988 Phys. Rev. B37 786

27. Bock C W and Trachtman M J 1994 Phys. Chem. 98 95

28. Newman A C D and Brown G 1974 Chemistry of clays and clay minerals (ed) A C D Newman, Monograph no. 5 (London: Mineral. Soc.) p 10

29. Chatterjee A, Iwasaki T, Ebina $\mathrm{T}$ and Miyamoto A 1999 Comput. Mater. Sci. 14119

30. Ransom B and Helgeson H 1995 Am. J. Sci. 295245

31. Bassett W A and Wu T C 1999 CMS Workshop Lectures, Synchrotron methods in clay science (eds) D G Schulze, J W Stucki and P M Bertsch (Boulder, CO: The Clay Mineral Soc.) pp 220-239 